\title{
INITIAL BOUNDARY VALUE PROBLEM FOR NONLINEAR DIRAC EQUATION OF GROSS-NEVEU TYPE IN $1+1$ DIMENSIONS
}

\author{
YONGQIAN ZHANG AND QIN ZHAO
}

\begin{abstract}
This paper studies an initial boundary value problem for a class of nonlinear Dirac equations with cubic terms and moving boundary. For the initial data with bounded $L^{2}$ norm and the suitable boundary conditions, the global existence and the uniqueness of the strong solution are proved.
\end{abstract}

\section{INTRODUCTION}

Consider the nonlinear Dirac equations

$$
\left\{\begin{array}{l}
i\left(u_{t}+u_{x}\right)=-m v+N_{1}(u, v), \\
i\left(v_{t}-v_{x}\right)=-m u+N_{2}(u, v),
\end{array}\right.
$$

in a domain $\Omega=\{(x, t) \mid t \geq 0, x \geq z(t)\}$ for $m \geq 0$ with initial data

$$
(u(x, t=0), v(x, t=0))=\left(u_{0}(x), v_{0}(x)\right), \quad x \geq 0,
$$

and boundary condition

$$
u(z(t), t)=\lambda(t) v(z(t), t), \quad t \geq 0 .
$$

The nonlinear terms take the following form

$$
\begin{gathered}
N_{1}=\partial_{\bar{u}} W(u, v)=\alpha u|v|^{2}+2 \beta v(\bar{u} v+u \bar{v}), \\
N_{2}=\partial_{\bar{v}} W(u, v)=\alpha v|u|^{2}+2 \beta u(\bar{u} v+u \bar{v}),
\end{gathered}
$$

with

$$
W(u, v)=\alpha|u|^{2}|v|^{2}+\beta(\bar{u} v+u \bar{v})^{2},
$$

where $\alpha, \beta \in R^{1}$ and $\bar{u}, \bar{v}$ are complex conjugate of $u$ and $v$.

The boundary $\{x=z(t)\}$, denoted by $\Gamma_{B}$, is assumed to satisfy the following,

(H1): $-1<z_{t}(t)<1$, for $t \geq 0$ and $z(0)=0$.

(H2): $|\lambda(t)|^{2}\left(1-z_{t}(t)\right) \leq\left(1+z_{t}(t)\right)$, for $t \geq 0$.

Key words and phrases. Nonlinear Dirac equation; Gross-Neveu model; global strong solution; Bony type functional; Glimm type functional.

( AMS subject classification. Primary: 35Q41 ; Secondary: 35L60, 35Q40) . 
Here and in sequel, we denote $z_{t}=\frac{d z}{d t}, \lambda_{t}=\frac{d \lambda}{d t}, u_{t}=\frac{\partial u}{\partial t}, u_{x}=\frac{\partial u}{\partial x}$ etc. for simplification.

The nonlinear Dirac equation (1.1) is called Thirring equation for $\alpha=1$ and $\beta=0$, while it is called Gross-Neveu equation for $\alpha=0$ and $\beta=1 / 4$; see for instance [23] and [14], 20]. There are a number of works devoted to the local and global well-poedness of the Cauchy problem for the nonlinear Dirac equation with various type of nonlinearities in different spatial dimensions (see for instance [2, 4, 6, 7, 9, 10, 11, 12, 14, 16, 20, 21, 23, 24, 25, and the references therein). There are also some papers on the initial boundary value problem (see for example [5] and [18]). In [5], motivated to study the Hawking effect describing the collapse of a spherically symmetric star to a Schwarzchild black hole, Bouvier and Gérard used technique from $C^{*}$ algebra to study the asymptotic behaviour of the global solution to (1.1), (1.2) and (1.3) with a class of special initial data in $R^{1+1}$, where the non-characteristic boundary is assumed to approach characteristic as $t \rightarrow \infty$, with $|\lambda(t)|^{2}\left(1-z_{t}(t)\right)=$ $\left(1+z_{t}(t)\right)$ for $t \geq 0$ and the solution is assumed to be bounded. In [18, 19], Naumkin proved the existence of global solution in $H^{1}$ to initial boundary value problem for Thirring model in quarter plane $\{t>0, x>0\}$ with small data and study the scattering behaviour of solution. To our knowledge there is no result on the well posedness of initial boundary value problem for Gross-Neveu model with general initial data in $L^{2}$. Our purpose is to prove the existence and the uniqueness in $C^{1}(\bar{\Omega})$ and in $L^{2}(\Omega)$ of global solution to (1.1-1.3).

The first result is the following.

Theorem 1.1. Suppose that (H1) and (H2) hold. Let $\left(u_{0}, v_{0}\right) \in C^{1}([0, \infty))$ with compact support in $[0, \infty)$ and satisfy the compatibility conditions as follow,

and

$$
u_{0}(0)=\lambda(0) v_{0}(0)
$$

$$
\begin{gathered}
\left(1-z_{t}(0)\right) u_{0 x}(0)+\lambda(0)\left(1+z_{t}(0)\right) v_{0 x}(0)+i \lambda(0)\left(m u_{0}(0)-N_{2}\left(u_{0}(0), v_{0}(0)\right)\right) \\
-i\left(m v_{0}(0)-N_{1}\left(u_{0}(0), v_{0}(0)\right)\right)+\lambda_{t}(0) v_{0}(0)=0 .
\end{gathered}
$$

Then (1.1-1.3) has a unique global solution $(u, v) \in C^{1}(\bar{\Omega})$.

This result could be generalized to the following case.

Theorem 1.2. Suppose that (H1) and (H2) hold. Let $\left(u_{0}, v_{0}\right) \in H^{1}([0, \infty))$ satisfy the compatibility conditions as follows,

$$
u_{0}(0)=\lambda(0) v_{0}(0) .
$$

Then (1.1] 1.3) has a unique global solution $(u, v) \in H_{l o c}^{1}(\Omega) \cap C(\bar{\Omega})$. Moreover,

$$
(u(\cdot, t), v(\cdot, t)) \in H^{1}([z(t), \infty))
$$

for $t \in[0, \infty)$. 
With Theorem 1.1, we can look for the global strong solution. Here the strong solution is defined as follows.

Definition 1.1. A pair of measurable functions $(u, v)$ is called a strong solution to (1.1]1.3) if there exits a sequence of classical solutions $\left(u^{(n)}, v^{(n)}\right) \in$ $C^{1}(\bar{\Omega})$ to (1.1) such that

$$
u^{(n)}(z(t), t)=\lambda(t) v^{(n)}(z(t), t), \quad \text { for } t>0,
$$

and

$$
\begin{gathered}
\lim _{n \rightarrow \infty} \int_{0}^{N}\left(\left|u^{(n)}(x, 0)-u_{0}\right|^{2}+\left|v^{(n)}(x, 0)-v_{0}\right|^{2}\right) d x=0, \\
\lim _{n \rightarrow \infty} \iint_{K}\left(\left|u^{(n)}-u\right|^{2}+\left|v^{(n)}-v\right|^{2}\right) d x d t=0
\end{gathered}
$$

for any compact set $K \subset \bar{\Omega}$ and for any $N>0$.

Theorem 1.3. Suppose that (H1) and (H2) hold. For any $\left(u_{0}, v_{0}\right) \in L_{l o c}^{2}([0, \infty))$, (1.1]1.3) has a unique global strong solution $(u, v) \in L_{l o c}^{2}(\Omega)$. Moreover, $|u||v| \in L_{l o c}^{2}(\Omega)$, and $(u, v)$ solves (1.1, 1.3) in the following sense,

$$
\begin{aligned}
& \iint_{\Omega}\left(i u\left(\phi_{t}+\phi_{x}\right)-m v \phi+N_{1}(u, v) \phi\right) d x d t=-i \int_{0}^{\infty} u_{0} \phi(x, 0) d x \\
& \iint_{\Omega}\left(i v\left(\psi_{t}-\psi_{x}\right)-m u \psi+N_{2}(u, v) \psi\right) d x d t=-i \int_{0}^{\infty} v_{0} \psi(x, 0) d x
\end{aligned}
$$

for any $(\phi, \psi) \in C^{1}(\bar{\Omega})$ with bounded support in $\bar{\Omega}$ and $(\phi, \psi)(z(t), t)=0$ for $t \geq 0$.

Moreover, we have the following.

Theorem 1.4. Suppose that (H1) and (H2) hold. If $\left(u_{0}, v_{0}\right) \in L^{2}([0, \infty))$, then the strong solution $(u, v)$ given by Theorem 1.3 satisfies the following,

$$
(u, v) \in L^{2}\left(\Omega \cap\left(R^{1} \times[0, T]\right)\right), \quad|u||v| \in L^{2}\left(\Omega \cap\left(R^{1} \times[0, T]\right)\right)
$$

for any $T>0$. Moreover, if $|\lambda(t)|^{2}\left(1-z_{t}(t)\right)=\left(1+z_{t}(t)\right)$ for $t \geq 0$, then

$$
\int_{z(t)}^{\infty}\left(|u(x, t)|^{2}+|v(x, t)|^{2}\right) d x=\int_{0}^{\infty}\left(\left|u_{0}(x)\right|^{2}+\left|v_{0}(x)\right|^{2}\right) d x
$$

for almost every $t \in[0, \infty)$.

The remaining is organized as follows. First, in section 2 , to prove Theorem 1.1 and Theorem 1.2 for $(1.1-1.3)$, we derive the equations (2.1) and (2.2) for $|u|^{2}$ and $|v|^{2}$ for local smooth solution $(u, v)$, and apply the characteristic method to the equations (2.1) and (2.2) to get the pointwise bounds on $|u|^{2}$ and $|v|^{2}$. Then it enables us to get the uniform $L^{\infty}$ bounds on $(u, v)$ in the 
domain $\Omega \cap\{0 \leq t<T\}$ for any $T>0$ and extend the local solution globally. In section 3 we introduce a Bony type functional $Q_{0}(t, \Delta)$ and a Glimm type functional $F_{0}(t, \Delta)=L(t, u, \Delta)+K_{0} L(t, v, \Delta)+C_{0} Q(t, \Delta)$ for smooth solution $(u, v)$ to get $L^{2}$ estimates of nonlinear term, $\iint_{\Delta}|u(x, t)|^{2}|v(x, t)|^{2} d x d t$ on each characteristic triangle $\Delta$. Here different from the work in [25], for the case that $\Delta \cap \partial \Omega \neq \emptyset$, by the assumption (H2) we choose a suitable constant $K_{0}>0$ so that the derivative of the weighted $L^{2}$ norm, $\frac{d}{d t}\left(L(t, u, \Delta)+K_{0} L(t, v, \Delta)\right)$ can control the possible increasing of the functional $Q_{0}(t, \Delta)$, and choose a suitable constant $C_{0}$ so that $F_{0}(t, \Delta)$ can control $\iint_{\Delta}|u(x, t)|^{2}|v(x, t)|^{2} d x d t$, while for the case that $\Delta \subseteq \Omega$ same argument as in [25] can be carried out to get the control on $\iint_{\Delta}|u(x, t)|^{2}|v(x, t)|^{2} d x d t$. In section 4, we consider the difference $(U, V)=\left(u-u^{\prime}, v-v^{\prime}\right)$ for two smooth solutions $(u, v)$ and $\left(u^{\prime}, v^{\prime}\right)$. We first write down the equations (4.1) and (4.2) for $(U, V)$, which contain $(U, V),(u, v)$ and $\left(u^{\prime}, v^{\prime}\right)$. Then we introduce a Bony type functional $Q_{1}(t, \Delta)$ and a Glimm type functional $F_{1}(t, \Delta)$ for $|U|^{2},|V|^{2},|u|^{2},|v|^{2}$ and $\left|u^{\prime}\right|^{2}$ and $\left|v^{\prime}\right|^{2}$, and use it to prove the $L^{2}$ stability estimates in Proposition 4.1. Here, as in section 3, for the case that $\Delta \cap \partial \Omega \neq \emptyset$, by the assumption (H2) we choose a suitable constant $K>0$ so that the derivative of the weighted $L^{2}$ norm, $\frac{d}{d t}\left(L(t, U, \Delta)+K_{1} L(t, V, \Delta)\right)$ can control the possible increasing of the functional $Q_{1}(t, \Delta)$. In section 5, we first approximate the initial data (1.2) by a sequence of smooth functions. Then, by the result on the global wellposedness for smooth solution in section 2, we can have a sequence of global smooth solutions for smooth data for (1.1). With the help of the $L^{2}$ stability estimates in section 4, we show that the sequence of global smooth solutions converges to a strong solution in $L^{2}(\Delta)$ for any triangle $\Delta$. In section 6. we complete the proof of Theorem 1.3 and Theorem 1.4.

\section{Global Classical solution}

For $T>0$, denote

$$
\Omega(T)=\{(x, t) \mid z(t) \leq x<\infty, 0 \leq t<T\} .
$$

Classical theory on semilinear hyperbolic systems [1] gives the following local existence result (see also [17]).

Lemma 2.1. Suppose that the compatibility conditions (1.6) and (1.7) hold. For any $\left(u_{0}, v_{0}\right) \in C^{1}([0, \infty))$ with compact support in $[0, \infty)$, there exists a $T_{*}>0$ such that $(1.1-1.3)$ has a unique solution $(u, v) \in C^{1}\left(\overline{\Omega\left(T_{*}\right)}\right)$.

Our aim in this section is to extend the solution $(u, v)$ globally to $\bar{\Omega}$. To this end, let $\left(u_{0}, v_{0}\right) \in C^{1}([0, \infty))$ with compact support and let $(u, v) \in C^{1}(\Omega(T))$ be the solution to (1.1-1.3) for $T \geq T_{*}$, taking $\left(u_{0}, v_{0}\right)$ as its initial data, we have to establish the estimates on $\|(u, v)\|_{L^{\infty}(\Omega(T))}$ in the next. Here we assume that the compatibility conditions (1.6) and (1.7) hold for $\left(u_{0}, v_{0}\right)$. 
Multiplying the first equation of (1.1) by $\bar{u}$ and the second equation by $\bar{v}$ gives

$$
\left(|u|^{2}\right)_{t}+\left(|u|^{2}\right)_{x}=2 m \Re(i \bar{u} v)+2 \Re\left(i \overline{N_{1}} u\right),
$$

and

$$
\left(|v|^{2}\right)_{t}-\left(|v|^{2}\right)_{x}=2 m \Re(i u \bar{v})+2 \Re\left(i \overline{N_{2}} v\right),
$$

which, together with the structure of nonlinear terms, leads to

$$
\left(|u|^{2}+|v|^{2}\right)_{t}+\left(|u|^{2}-|v|^{2}\right)_{x}=0
$$

For the nonlinear terms in the righthand side of (2.1) and (2.2), we have the following by direct computation.

Lemma 2.2. Let $r_{0}(x, t)=m\left(|u(x, t)|^{2}+|v(x, t)|^{2}\right)+8|\beta||u(x, t)|^{2}|v(x, t)|^{2}$. Then there hold the followings,

$$
\left|2 m \Re(i \bar{u} v)+2 \Re\left(i \overline{N_{1}} u\right)\right| \leq r_{0}(x, t)
$$

and

$$
\left|2 m \Re(i \bar{v} u)+2 \Re\left(i \overline{N_{2}} v\right)\right| \leq r_{0}(x, t) .
$$

And we have the estimates on the $L^{2}$ norm of the solution as follows.

Lemma 2.3. Let $E_{0}=\int_{0}^{\infty}\left(\left|u_{0}(x)\right|^{2}+\left|v_{0}(x)\right|^{2}\right) d x$. Then for any $t \in[0, T)$, there holds the following,

$$
\int_{z(t)}^{\infty}\left(|u(x, t)|^{2}+|v(x, t)|^{2}\right) d x \leq E_{0} .
$$

Proof. By (1.3) and (2.3), and by assumption (H2), we have

$$
\begin{aligned}
& \frac{d}{d t} \int_{z(t)}^{\infty}\left(|u(x, t)|^{2}+|v(x, t)|^{2}\right) d x \\
= & |u(z(t), t)|^{2}\left(1-z_{t}(t)\right)-|v(z(t), t)|^{2}\left(1+z_{t}(t)\right) \\
= & |v(z(t), t)|^{2}\left[|\lambda(t)|^{2}\left(1-z_{t}(t)\right)-\left(1+z_{t}(t)\right)\right] \leq 0,
\end{aligned}
$$

which gives the desired inequality and completes the proof.

We consider the characteristic triangles for $(u, v)$ in $\Omega(T)$. For any $a, b \in R^{1}$ with $a<b$ and for any $t_{0} \geq 0$, we denote

$$
\Delta\left(a, b, t_{0}\right)=\left\{(x, t) \mid a-t_{0}+t<x<b+t_{0}-t, t_{0}<t<\frac{b-a}{2}+t_{0}\right\},
$$

see Figure 1, and, denote

$$
\Gamma_{u}\left(x_{0}, t_{0} ; t_{1}\right)=\left\{(x, t) \mid x=x_{0}-t_{0}+t, t_{1} \leq t \leq t_{0}\right\}
$$

and

$$
\Gamma_{v}\left(x_{0}, t_{0} ; t_{1}\right)=\left\{(x, t) \mid x=x_{0}+t_{0}-t, t_{1} \leq t \leq t_{0}\right\}
$$

for $t_{1} \leq t_{0}$, see Figure 2. It is obvious that $\Gamma_{u}\left(x_{0}, t_{0} ; t_{1}\right)$ is a characteristic line 

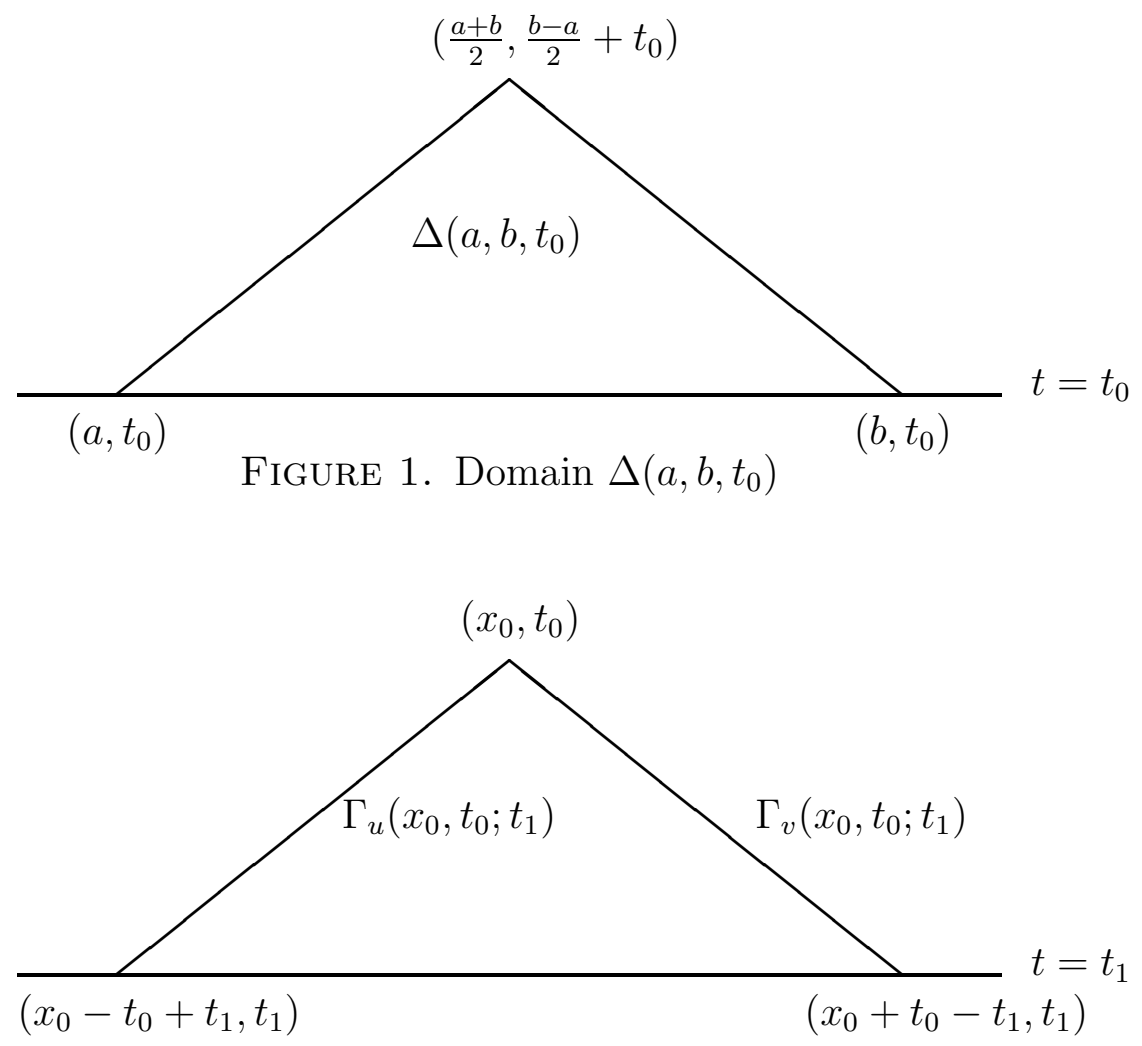

Figure 2. Characteristic lines $\Gamma_{u}$ and $\Gamma_{v}$

for the first equation of $u$ in (1.1) while $\Gamma_{v}\left(x_{0}, t_{0} ; t_{1}\right)$ is a characteristic line for the second equation of $v$ in (1.1).

Along these characteristic lines in $\Omega(T)$, we have the following estimates.

Lemma 2.4. If $\Gamma_{v}\left(x_{0}, t_{0} ; t_{1}\right) \subseteq \overline{\Omega(T)}$, then

$$
\int_{t_{1}}^{t_{0}}\left|u\left(x_{0}+t_{0}-s, s\right)\right|^{2} d s \leq E_{0} .
$$

Here $E_{0}=\int_{0}^{\infty}\left(\left|u_{0}\right|^{2}+\left|v_{0}\right|^{2}\right) d x$.

Proof. Denote

$$
\omega\left(x_{0}, t_{0}\right)=\left\{(x, t) \mid z(t) \leq x \leq x_{0}+t_{0}-t, 0 \leq t \leq t_{0}\right\}
$$


Then taking the integration of (2.3) over $\omega\left(x_{0}, t_{0}\right)$ gives the following,

$$
\begin{aligned}
& \int_{0}^{x_{0}+t_{0}}\left(\left|u_{0}(x)\right|^{2}+\left|v_{0}(x)\right|^{2}\right) d x \\
= & 2 \int_{0}^{t_{0}}\left|u\left(x_{0}+t_{0}-s, s\right)\right|^{2} d s+\int_{z\left(t_{0}\right)}^{x_{0}}\left(\left|u\left(x, t_{0}\right)\right|^{2}+\left|v\left(x, t_{0}\right)\right|^{2}\right) d x \\
& +\int_{0}^{t_{0}}\left\{\left(-1+z_{t}(s)\right)|u(z(s), s)|^{2}+\left(1+z_{t}(s)\right)|v(z(s), s)|^{2}\right\} d s \\
\geq & \int_{0}^{t_{0}}\left|u\left(x_{0}+t_{0}-s, s\right)\right|^{2} d s,
\end{aligned}
$$

where we use the boundary condition (1.3) and assumption (H2) to get the last inequality. This implies the result and the proof is complete.

Lemma 2.5. If $\Gamma_{u}\left(x_{0}, t_{0} ; t_{1}\right) \subseteq \overline{\Omega(T)}$, then

$$
\int_{t_{1}}^{t_{0}}\left|v\left(x_{0}-t_{0}+s, s\right)\right|^{2} d s \leq E_{0} .
$$

Here $E_{0}=\int_{0}^{\infty}\left(\left|u_{0}\right|^{2}+\left|v_{0}\right|^{2}\right) d x$.

Proof. Since $\Gamma_{u}\left(x_{0}, t_{0} ; t_{1}\right) \subseteq \overline{\Omega(T)}$, then the domain

$$
\Delta\left(x_{0}-t_{0}+t_{1}, x_{0}+t_{0}-t_{1}, t_{1}\right) \subseteq \overline{\Omega(T)} .
$$

Taking the integration of (2.3) over $\Delta\left(x_{0}-t_{0}+t_{1}, x_{0}+t_{0}-t_{1}, t_{1}\right)$, we have

$$
\begin{aligned}
\int_{t_{1}}^{t_{0}}\left|v\left(x_{0}-t_{0}+s, s\right)\right|^{2} d s & \leq \int_{x_{0}-t_{0}+t_{1}}^{x_{0}+t_{0}-t_{1}}\left(\left|u\left(x, t_{1}\right)\right|^{2}+\left|v\left(x, t_{1}\right)\right|^{2}\right) d x \\
& \leq \int_{z\left(t_{1}\right)}^{\infty}\left(\left|u\left(x, t_{1}\right)\right|^{2}+\left|v\left(x, t_{1}\right)\right|^{2}\right) d x \\
& \leq E_{0}
\end{aligned}
$$

where the last inequality is given by Lemma 2.3 . The proof is complete.

Using the above estimates on along the characteristic lines, we can get the following pointwise estimates on $v$ at first.

Lemma 2.6. For $(x, t) \in \overline{\Omega(T)}$,

$$
|v(x, t)|^{2} \leq\left(\left|v_{0}(x+t)\right|^{2}+m E_{0}\right) \exp \left(m t+8|\beta| E_{0}\right) \text {. }
$$

Here $E_{0}=\int_{0}^{\infty}\left(\left|u_{0}\right|^{2}+\left|v_{0}\right|^{2}\right) d x$.

Proof. Assumption (H1) implies that

$$
\Gamma_{v}(x, t ; 0) \subset \overline{\Omega(T)}
$$

for any $(x, t) \in \overline{\Omega(T)}$. 
Then, by Lemma 2.2, along $\Gamma_{v}(x, t ; 0)$ we use the equation (2.2) to derive that

$\frac{d}{d s}|v(x+t-s, s)|^{2} \leq m|u(x+t-s, s)|^{2}+\left(m+8|\beta||u(x+t-s, s)|^{2}\right)|v(x+t-s, s)|^{2}$.

Therefore

$$
\begin{aligned}
\frac{d}{d s}\left(|v(x+t-s, s)|^{2} e_{1}(x, t, s)\right) & \leq m|u(x+t-s, s)|^{2} e_{1}(x, t, s) \\
& \leq m|u(x+t-s, s)|^{2}
\end{aligned}
$$

where

$$
e_{1}(x, t, s)=\exp \left(-m s-8|\beta| \int_{0}^{s}|u(x+t-\tau, \tau)|^{2} d \tau\right) .
$$

Taking the integration of the above from $s=0$ to $t$, we can prove the desired result by Lemma 2.4. The proof is complete.

To get the pointwise estimates on $u$, we look for the intersection point of the boundary $\Gamma_{B}$ and the characteristic line $\{(x, t) \mid x-t=b\}$ for $b \leq 0$.

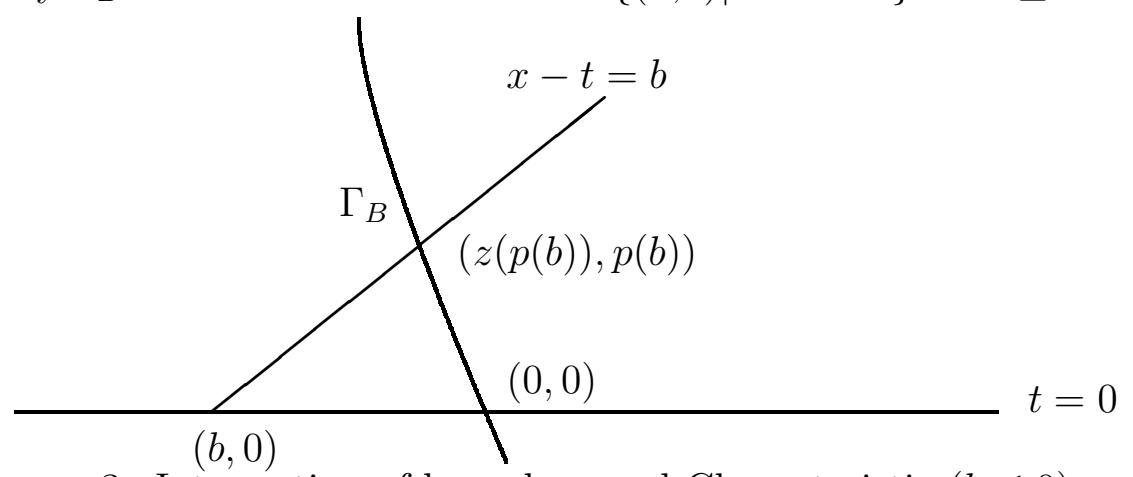

FiguRE 3. Intersection of boundary and Characteristic $(b \leq 0)$

Lemma 2.7. For any $b \leq 0$, the equation $z(t)-t=b$ has a unique solution $t=p(b)$, where $p \in C^{1}(-\infty, 0]$ and $p^{\prime}(s)<0$ for $s \leq 0$.

Proof. From assumption (H1) it follows that

$$
z_{t}(t)-1<0
$$

for $t>0$, which implies that the function $z(t)-t$ has a global inverse $p \in$ $C^{1}(-\infty, 0]$. Moreover,

$$
p^{\prime}(s)=\frac{1}{1-z_{t}(p(s))}<0 .
$$

Therefore the proof is complete.

Now we can have the following pointwise estimates on $u$. 
Lemma 2.8. If $(x, t) \in \overline{\Omega(T)}$ with $x-t \geq 0$, then

$$
|u(x, t)|^{2} \leq\left(\left|u_{0}(x-t)\right|^{2}+m E_{0}\right) \exp \left(m t+8|\beta| E_{0}\right) .
$$

If $(x, t) \in \overline{\Omega(T)}$ with $x-t<0$, then

$$
|u(x, t)|^{2} \leq\left(|\lambda(t)|^{2}+1\right)\left(\left|v_{0}(2 p(x-t)+x-t)\right|^{2}+m E_{0}\right) \exp \left(2 m t+16|\beta| E_{0}\right) .
$$

Here $E_{0}=\int_{0}^{\infty}\left(\left|u_{0}\right|^{2}+\left|v_{0}\right|^{2}\right) d x$.

Proof. For $(x, t) \in \overline{\Omega(T)}$ with $x-t \geq 0$, the assumption (H1) implies that

$$
\Gamma_{u}(x, t ; 0) \subseteq \overline{\Omega(T)} .
$$

Then, by (2.1) and by Lemma 2.2, we have

$$
\begin{aligned}
\frac{d}{d s}\left(|u(x-t+s, s)|^{2} e_{2}(x, t, s)\right) & \leq m|v(x-t+s, s)|^{2} e_{2}(x, t, s) \\
& \leq m|v(x-t+s, s)|^{2}
\end{aligned}
$$

where

$$
e_{2}(x, t, s)=\exp \left(-m s-8|\beta| \int_{0}^{s}|v(x-t+\tau, \tau)|^{2} d \tau\right)
$$

Taking the integration of (2.5) from 0 to $t$ and using Lemma 2.5, we get

$$
|u(x, t)|^{2} \leq\left(\left|u_{0}(x-t)\right|^{2}+m E_{0}\right) \exp \left(m t+8|\beta| E_{0}\right) .
$$

For $(x, t) \in \overline{\Omega(T)}$ with $x-t<0$, Lemma 2.7 implies that the characteristic line $\Gamma_{u}(x, t ; 0)$ and the boundary intersect only at the point $(z(p(x-t)), p(x-$ $t)$ ).

Then, by (2.1) and by Lemma 2.2, along the characteristic line $\Gamma_{u}(x, t ; p(x-$ $t)$ ) we have

$$
\begin{aligned}
\frac{d}{d s}\left(|u(x-t+s, s)|^{2} e_{3}(x, t, s)\right) & \leq m|v(x-t+s, s)|^{2} e_{3}(x, t, s) \\
& \leq m|v(x-t+s, s)|^{2}
\end{aligned}
$$

where

$$
e_{3}(x, t, s)=\exp \left(-m(s-p(x-t))-8|\beta| \int_{p(x-t)}^{s}|v(x-t+\tau, \tau)|^{2} d \tau\right) .
$$

Taking the integration of the above from $p(x-t)$ to $t$, we use Lemma 2.5 and Lemma 2.6 to get the following,

$$
\begin{aligned}
|u(x, t)|^{2} & \leq\left(|u(p(x-t)+x-t, p(x-t))|^{2}+m E_{0}\right) \exp \left(m t+8|\beta| E_{0}\right) \\
& \leq\left(|\lambda(t)|^{2}|v(p(x-t)+x-t, p(x-t))|^{2}+m E_{0}\right) \exp \left(m t+8|\beta| E_{0}\right) \\
& \leq\left(|\lambda(t)|^{2}+1\right)\left(\left|v_{0}(2 p(x-t)+x-t)\right|^{2}+m E_{0}\right) \exp \left(2 m t+16|\beta| E_{0}\right) .
\end{aligned}
$$

The proof is complete.

Now using the pointwise estimates on $u$ and $v$, we can prove Theorem 1.1 . 
Proof of Theorem 1.1. For $\left(u_{0}, v_{0}\right) \in H^{1}([0, \infty)) \subset L^{\infty}([0, \infty))$, Lemma 2.6 and Lemma 2.8 lead to

$|u(x, t)|^{2}+|v(x, t)|^{2} \leq\left(|\lambda(t)|^{2}+2\right)\left(||\left(u_{0}, v_{0}\right) \|_{L^{\infty}}+2 m E_{0}\right) \exp \left(2 m t+16|\beta| E_{0}\right)$

for $x \geq z(t)$ and $0 \leq t<T$.

Then by the standard theory on semilinear hyperbolic equations (see [1] for instance), we can extend the solution $(u, v)$ across the time $t=T$.

Therefore, repeating the same argument for any time, we can extend the solution globally to $\bar{\Omega}$. The proof is complete.

Furthermore Theorem 1.2 follows from Theorem 1.1.

Proof of Theorem 1.2. Let $\left(\phi_{0}, \psi_{0}\right) \in C_{c}^{\infty}\left(R^{1}\right)$ be a pair of functions such that $\phi_{0}(x)=u_{0}(0)$ and $\psi_{0}(x)=v_{0}(0)$ for $x$ belonging to a neighbourhood of zero. Then we choose a sequence of functions $\left(\phi_{1}^{k}, \psi_{1}^{k}\right) \in C_{c}^{\infty}(0, \infty)$ such that $\left(u_{0}^{(k)}, v_{0}^{(k)}\right):=\left(\phi_{0}+\phi_{1}^{k}, \psi_{0}+\psi_{1}^{k}\right)$ is convergent to $\left(u_{0}, v_{0}\right)$ in $H^{1}(0, \infty)$ as $k$ tends to $\infty$.

It is obvious that $\left(u_{0}^{(k)}, v_{0}^{(k)}\right)$ satisfies the compatibility conditions as (1.6) and (1.7). Therefore, by Theorem 1.1, the equations (1.1) has a global smooth solution $\left(u^{(k)}, v^{(k)}\right)$ with the initial data $\left(u_{0}^{(k)}, v_{0}^{(k)}\right)$ for $k \geq 1$.

Moreover, by Lemma 2.6 and Lemma 2.8, we have

$$
\sup _{k \geq 1}\left\|\left(u^{(k)}, v^{(k)}\right)\right\|_{L^{\infty}(\Omega(T))}<\infty
$$

for any $T>0$, which enables us to show as in [1] and [17] that the sequence $\left(u^{(k)}, v^{(k)}\right)$ is convergent in $H^{1}(\Omega(T))$ to a solution $(u, v)$ of (1.1)-(1.3) as $k$ tends to $\infty$ for any $T>0$.

The uniqueness can be proved by the the energy inequality for the difference of solutions in $L^{\infty}(\Omega(T)) \cap H^{1}(\Omega(T))$ as in [1] and [17]. The proof is complete.

\section{Estimates On THE Classical SOLUTiON}

Consider the case that $\left(u_{0}, v_{0}\right) \in C^{1}([0, \infty))$, and let $(u, v) \in C^{1}(\bar{\Omega})$ be the global solution to (1.1) with boundary condition (1.3). Here we assume that the compatibility condition (1.6) and (1.7) hold. Our aim in this section is to establish the local estimates on $(u, v)$.

To this end, set $\Delta=\Delta\left(a, b ; t_{0}\right)$ for simplification and assume that $\Delta \cap \Omega \neq \emptyset$ in this section.

Let

$$
x_{0}^{\prime}=\frac{b+a}{2}, t_{0}^{\prime}=\frac{b-a}{2}+t_{0} .
$$

Then $\Gamma_{u}\left(x_{0}^{\prime}, t_{0}^{\prime} ; t_{0}\right)$ and $\Gamma_{v}\left(x_{0}^{\prime}, t_{0}^{\prime} ; t_{0}\right)$ are the left and right edges of $\Delta$. By $(\mathrm{H} 1)$, $\Gamma_{B}$ and $\Gamma_{u}\left(x_{0}^{\prime}, t_{0}^{\prime} ; t_{0}\right) \cup \Gamma_{v}\left(x_{0}^{\prime}, t_{0}^{\prime} ; t_{0}\right)$ intersect at one point at the most. 
We introduce a time interval as follows. Denote

$$
I_{\Delta}=\left\{t \mid t_{0} \leq t \leq \frac{b-a}{2}+t_{0}, z(t) \leq b+t_{0}-t\right\} .
$$

By Lemma 2.7, we have the following.

Lemma 3.1. There hold the following statements. (1) If $\Gamma_{B} \cap \Gamma_{v}\left(x_{0}^{\prime}, t_{0}^{\prime} ; t_{0}\right)=$ $\left\{\left(z\left(\tau_{1}\right), \tau_{1}\right)\right\}$ for some $\tau_{1} \geq t_{0}$ (Figure 4), then $I_{\Delta}=\left[t_{0}, \tau_{1}\right]$ and

$$
\{x \mid(x, t) \in \overline{\Delta \cap \Omega}\}=\left[z(t), b+t_{0}-t\right] .
$$

(2) If $\Gamma_{B} \cap \Gamma_{u}\left(x_{0}^{\prime}, t_{0}^{\prime} ; t_{0}\right)=\left\{\left(z\left(\tau_{2}\right), \tau_{2}\right)\right\}$ for some $\tau_{2} \geq t_{0}$ (Figure [5), then $I_{\Delta}=\left[t_{0}, \frac{b-a}{2}+t_{0}\right]$ and

$$
\{x \mid(x, t) \in \overline{\Delta \cap \Omega}\}=\left[z(t), b+t_{0}-t\right] \text { fort } \in\left[t_{0}, \tau_{2}\right],
$$

and

$$
\{x \mid(x, t) \in \overline{\Delta \cap \Omega}\}=\left[a-t_{0}+t, b+t_{0}-t\right] \text { fort } \in\left[\tau_{2}, \frac{b-a}{2}+t_{0}\right] .
$$

(3) If $\Delta \subset \Omega$, then $I_{\Delta}=\left[t_{0}, \frac{b-a}{2}+t_{0}\right]$ and

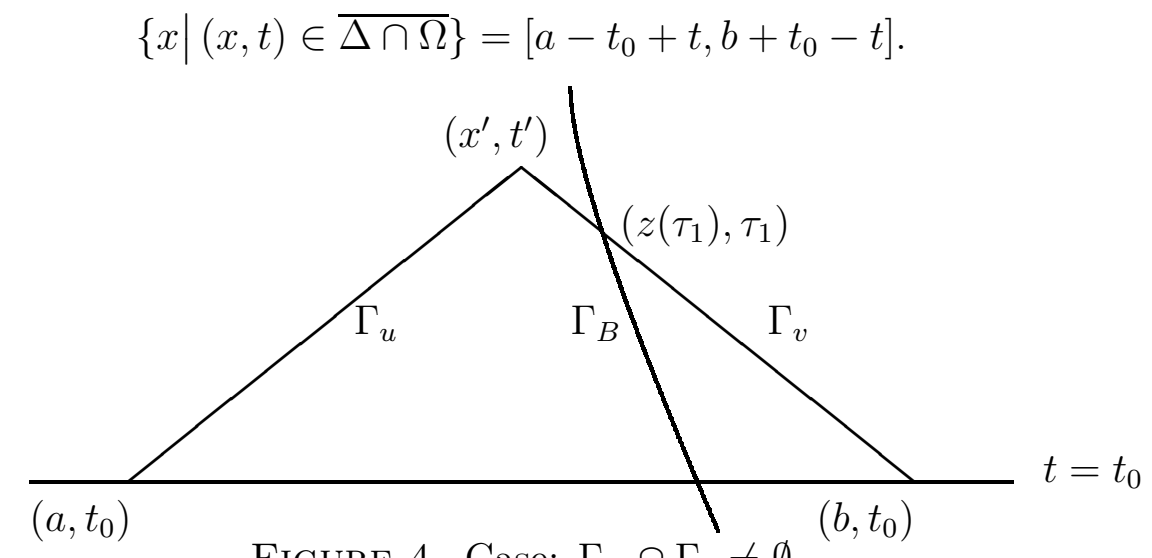

Figure 4. Case: $\Gamma_{B} \cap \Gamma_{v} \neq \emptyset$

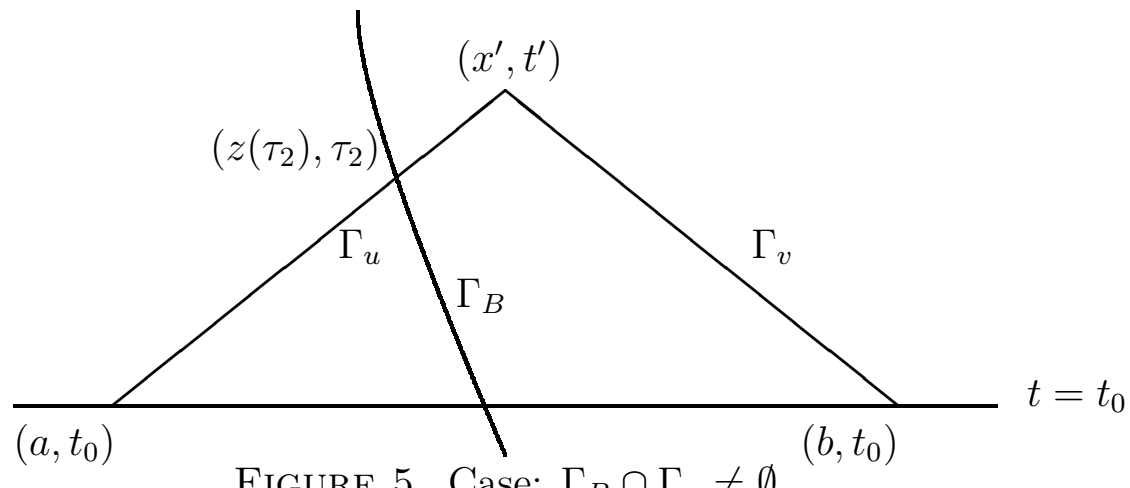

Figure 5. Case: $\Gamma_{B} \cap \Gamma_{u} \neq \emptyset$

Now we can define the functionals for $(u, v)$ on $\Delta \cap \Omega$ as follow. 
Definition 3.1. For $t \in I_{\Delta}$, and for any $w \in C^{1}(\bar{\Omega})$, define,

$$
L(t, w, \Delta)=\int_{z_{a}(t)}^{b-t+t_{0}}|w(x, t)|^{2} d x,
$$

where

$$
z_{a}(t)=\max \left\{a+t-t_{0}, z(t)\right\} .
$$

Definition 3.2. For $t \in I_{\Delta}$, and for the solution $(u, v)$, define

$$
L_{0}(t, \Delta)=L(t, u, \Delta)+L(t, v, \Delta)
$$

and

$$
\begin{gathered}
D_{0}(t, \Delta)=\int_{z_{a}(t)}^{b-t+t_{0}}|u(x, t)|^{2}|v(x, t)|^{2} d x \\
Q_{0}(t, \Delta)=\iint_{z_{a}(t)<x<y<b-t+t_{0}}|u(x, t)|^{2}|v(y, t)|^{2} d x d y
\end{gathered}
$$

where

$$
z_{a}(t)=\max \left\{a+t-t_{0}, z(t)\right\} .
$$

Then we have the following estimates on the $L^{2}$ norm.

Lemma 3.2. For $t \in I_{\Delta}$, there holds the following,

$$
L_{0}(t, \Delta) \leq L_{0}\left(t_{0}, \Delta\right)
$$

Proof. It suffices to prove lemma for three cases according to Lemma 3.1.

Case 1: The right edge of $\Delta$ and $\Gamma_{B}$ intersect at some point $\left(z\left(\tau_{1}\right), \tau_{1}\right)$, see Figure 4, In this case $I_{\Delta}=\left[t_{0}, \tau_{1}\right]$.

Then for $t \in\left[t_{0}, \tau_{1}\right], z_{a}(t)=z(t)$. Moreover, by (1.3) and (2.3), and by assumption (H2), we have

$$
\begin{aligned}
& \frac{d}{d t} \int_{z(t)}^{b-t+t_{0}}\left(|u(x, t)|^{2}+|v(x, t)|^{2}\right) d x \\
= & |u(z(t), t)|^{2}\left(1-z_{t}(t)\right)-|v(z(t), t)|^{2}\left(1+z_{t}(t)\right) \\
& -\left(\left|u\left(b-t+t_{0}, t\right)\right|^{2}+\left|v\left(b-t+t_{0}, t\right)\right|^{2}\right) \\
\leq & |v(z(t), t)|^{2}\left[|\lambda(t)|^{2}\left(1-z_{t}(t)\right)-\left(1+z_{t}(t)\right)\right] \leq 0 .
\end{aligned}
$$

This leads to the desired result.

Case 2: The left edge of $\Delta$ and $\Gamma_{B}$ intersect at some point $\left(z\left(\tau_{2}\right), \tau_{2}\right)$, see Figure 5, Then $I_{\Delta}=\left[t_{0}, t_{0}+\frac{b-a}{2}\right]$.

For $t \in\left[t_{0}, \tau_{2}\right], z_{a}(t)=z(t)$, and in the same way as in the proof of Case 1 , we can get

$$
L_{0}(t, \Delta) \leq L_{0}\left(t_{0}, \Delta\right)
$$


For $t \in\left[\tau_{2}, \frac{b-a}{2}+t_{0}\right], z_{a}(t)=a-t_{0}+t$, then we can use the result for Case 2 to deduce that

$$
L_{0}(t, \Delta) \leq L_{0}\left(\tau_{2}, \Delta\right)
$$

Case 3: $\Delta$ lies in the interior of $\Omega$. The proof can be carried out in the same way as in Case 1.

Therefore the proof is complete.

For any $T>0$, we recall the notation

$$
\Omega(T)=\{(x, t) \mid z(t) \leq x<\infty, 0 \leq t<T\},
$$

and have the control on the potential $Q_{0}$ for the case that $\Delta \subset \Omega(T)$ as follows.

Lemma 3.3. Suppose that $\Delta \subset \Omega(T)$ for $T>0$. Then there exists constants $\delta_{0}>0$ such that for the initial data satisfying $L_{0}\left(t_{0}, \Delta\right) \leq \delta_{0}$ there holds the following

$$
\frac{d Q_{0}(t, \Delta)}{d t}+D_{0}(t, \Delta) \leq 2 m\left(L_{0}\left(t_{0}, \Delta\right)\right)^{2}
$$

for $t \in\left(t_{0}, \frac{b-a}{2}+t_{0}\right)$. Therefore,

$$
\begin{aligned}
Q_{0}(t, \Delta)+\int_{t_{0}}^{t} D_{0}(\tau, \Delta) d \tau & \leq 2 m\left(L_{0}\left(t_{0}, \Delta\right)\right)^{2}\left(t-t_{0}\right)+Q_{0}\left(t_{0}, \Delta\right) \\
& \leq 2 m\left(L_{0}\left(t_{0}, \Delta\right)\right)^{2}\left(t-t_{0}\right)+\left(L_{0}\left(t_{0}, \Delta\right)\right)^{2}
\end{aligned}
$$

for $t \in\left[t_{0}, \frac{b-a}{2}+t_{0}\right]$. Here $\delta_{0}$ is independent of $T$.

The proof of Lemma 3.3 has been given in [25] and is similar to the proof of Lemma 3.4 in the next.

To get the control on the potential $Q_{0}$ near the boundary, we introduce a new functional as follows.

Definition 3.3. For constants $K_{0}>0$ and $C_{0}>0$ and for $t \in I_{\Delta}$, define

$$
F_{0}(t, \Delta)=L(t, u, \Delta)+K_{0} L(t, v, \Delta)+C_{0} Q_{0}(t, \Delta) .
$$

For any $T>0$, we have the control on $F_{0}$ near the boundary as follows.

Lemma 3.4. Suppose that $\Delta \subset R^{1} \times[0, T]$ and $\Delta \cap \Gamma_{B} \neq \emptyset$ for $T>0$. Then there exist constants $\delta_{0}>0, K_{0}>0$ and $C_{0}>0$ such that for $L_{0}\left(t_{0}, \Delta\right) \leq \delta_{0}$ there hold the following,

$$
\frac{d}{d t} F_{0}(t, \Delta) \leq-D_{0}(t, \Delta)-\left|v\left(z_{a}(t), t\right)\right|^{2}+O(1) \delta_{0},
$$

for $t \in I_{\Delta}$ with $z(t) \neq a+t-t_{0}$. Here the constants $\delta_{0}, K_{0}$ and $C_{0}$ depend only on $T$; and the bound of $O(1)$ depends only on $T$. 
Proof. For simplification, we denote $L_{0}(t, \Delta), D_{0}(t, \Delta), F_{0}(t, \Delta)$ and $Q_{0}(t, \Delta)$ by $L_{0}(t), D_{0}(t), F_{0}(t)$ and $Q_{0}(t)$. Now it suffices to prove the lemma for two cases.

Case 1: The boundary $\Gamma_{B}$ and the right edge $\Gamma_{v}$ of $\Delta$ intersect at the point $\left(z\left(\tau_{1}\right), \tau_{1}\right)$ for some $\tau_{1} \in\left[t_{0}, t_{0}+\frac{b-a}{2}\right]$, see Figure 4 .

Then $I_{\Delta}=\left[t_{0}, \tau_{1}\right], z_{a}(t)=z(t)$. For $t \in\left[t_{0}, \tau_{1}\right]$, by Lemma 2.2, we use (2.1), (2.2) to get

$$
\begin{aligned}
\frac{d}{d t} L(t, u, \Delta) \leq & \int_{z(t)}^{b-t+t_{0}}\left(-\left(|u(x, t)|^{2}\right)_{x}+r_{0}(x, t)\right) d x \\
& -\left|u\left(b-t+t_{0}, t\right)\right|^{2}-z_{t}(t)|u(z(t), t)|^{2} \\
\leq & \left(1-z_{t}(t)\right)|u(z(t), t)|^{2}+\int_{z(t)}^{b-t+t_{0}} r_{0}(x, t) d x
\end{aligned}
$$

and

$$
\begin{aligned}
\frac{d}{d t} L(t, v, \Delta) \leq & \int_{z(t)}^{b-t+t_{0}}\left(\left(|v(x, t)|^{2}\right)_{x}+r_{0}(x, t)\right) d x \\
& -\left|v\left(b-t+t_{0}, t\right)\right|^{2}-z_{t}(t)|v(z(t), t)|^{2} \\
\leq & -\left(1+z_{t}(t)\right)|v(z(t), t)|^{2}+\int_{z(t)}^{b-t+t_{0}} r_{0}(x, t) d x
\end{aligned}
$$

which lead to

$$
\begin{aligned}
\frac{d}{d t}\left(L(t, u, \Delta)+K_{0} L(t, v, \Delta)\right) \leq & \left(\left(1-z_{t}(t)\right)|\lambda(t)|^{2}-K_{0}\left(1+z_{t}(t)\right)\right)|v(z(t), t)|^{2} \\
& +\left(1+K_{0}\right) \int_{z(t)}^{b-t+t_{0}} r_{0}(x, t) d x \\
\leq & -2|v(z(t), t)|^{2}+\left(1+K_{0}\right) \int_{z(t)}^{b-t+t_{0}} r_{0}(x, t) d x \\
\leq & -2|v(z(t), t)|^{2}+O(1)\left(L_{0}(t)+D_{0}(t)\right),
\end{aligned}
$$

where we choose $K_{0}>1$ large enough so that

$$
\left(1-z_{t}(t)\right)|\lambda(t)|^{2}-K_{0}\left(1+z_{t}(t)\right)<-2
$$

for $t \in[0, T]$.

On the other hand, by Lemma 2.2, we use (2.1), (2.2) again to get the following for $Q_{0}$, 


$$
\begin{aligned}
\frac{d}{d t} Q_{0}(t)= & \iint_{z(t)<x<y<b-t+t_{0}}\left(\left(|u(x, t)|^{2}\right)_{t}|v(y, t)|^{2}+|u(x, t)|^{2}\left(|v(y, t)|^{2}\right)_{t}\right) d x d y \\
& +\left.\left(\frac{d}{d t} \iint_{z_{a}(t)<x<y<b-t+t_{0}}|u(x, s)|^{2}|v(y, s)|^{2} d x d y\right)\right|_{s=t} \\
\leq & \int_{z(t)}^{b-t+t_{0}}\left(|u(z(t), t)|^{2}-|u(y, t)|^{2}\right)|v(y, t)|^{2} d y \\
& +\int_{z(t)}^{b-t+t_{0}}|u(x, t)|^{2}\left(\left|v\left(b-t+t_{0}, t\right)\right|^{2}-|v(x, t)|^{2}\right) d x \\
& +\int_{z(t)}^{b-t+t_{0}} r_{0}(x, t) d x \int_{z(t)}^{b-t+t_{0}}\left(|u(y, t)|^{2}+|v(y, t)|^{2}\right) d y \\
& -z_{t}(t) \int_{z(t)}^{b-t+t_{0}}|u(z(t), t)|^{2}|v(y, t)|^{2} d y \\
& -\int_{z(t)}^{b-t+t_{0}}|u(x, t)|^{2}\left|v\left(b-t+t_{0}, t\right)\right|^{2} d x \\
\leq & \left(-2+O(1) L_{0}(t)\right) \int_{z(t)}^{b-t+t_{0}}|u(x, t)|^{2}|v(x, t)|^{2} d x \\
& +\left(1-z_{t}(t)\right)|u(z(t), t)|^{2} \int_{z(t)}^{b-t+t_{0}}|v(y, t)|^{2} d y+O(1)\left(L_{0}(t)\right)^{2} .
\end{aligned}
$$

Therefore,

$$
\begin{aligned}
\frac{d}{d t} F_{0}(t) \leq & -D_{0}(t)\left(\left(2-O(1) L_{0}(t)\right) C_{0}-O(1)\right) \\
& -|v(z(t), t)|^{2}\left(2-C_{0}\left(1-z_{t}(t)\right)|\lambda(t)|^{2} L_{0}(t)\right) \\
& +L_{0}(t)\left(O(1)+O(1) C_{0} L_{0}(t)\right) \\
\leq & -D_{0}(t)-|v(z(t), t)|^{2}+O(1) L_{0}(t) \\
\leq & -D_{0}(t)-|v(z(t), t)|^{2}+O(1) L_{0}\left(t_{0}\right)
\end{aligned}
$$

where we choose constant $C_{0}>0$ and $\delta_{0}$ such that $L_{0}\left(t_{0}\right) \leq \delta_{0}$ and

$$
\begin{gathered}
\left(2-O(1) \delta_{0}\right) C_{0}-O(1) \geq 1, \\
2-C_{0}\left(1-z_{t}(t)\right)|\lambda(t)|^{2} \delta_{0} \geq 1
\end{gathered}
$$

for $t \in[0, T]$. Then (3.4) is proved for this case.

Case 2: The boundary $\Gamma_{B}$ and the right edge $\Gamma_{v}$ of $\Delta$ intersect at the point $\left(z\left(\tau_{2}\right), \tau_{2}\right)$ for some $\tau_{2} \in\left[t_{0}, t_{0}+\frac{b-a}{2}\right]$, see Figure 5. The proof of (3.4) can be carried out in the same way as in Case 1 for $t \neq \tau_{2}$. Thus the proof is complete. 
4. Estimates on the Difference Between the Classical SOlutions

Let $(u, v) \in C^{1}(\bar{\Omega})$ and $\left(u^{\prime}, v^{\prime}\right) \in C^{1}(\bar{\Omega})$ be two classical solutions to (1.1) with (1.3). We consider the difference between these two solutions and denote

$$
(U, V)=\left(u-u^{\prime}, v-v^{\prime}\right) \text {. }
$$

Then,

$$
\begin{aligned}
U_{t}+U_{x} & =i m V-i\left(N_{1}(u, v)-N_{1}\left(u^{\prime}, v^{\prime}\right)\right), \\
V_{t}-V_{x} & =i m U-i\left(N_{2}(u, v)-N_{2}\left(u^{\prime}, v^{\prime}\right)\right),
\end{aligned}
$$

which lead to

$$
\begin{aligned}
& \left(|U|^{2}\right)_{t}+\left(|U|^{2}\right)_{x}=\Re 2\left\{i m V \bar{U}-i\left(N_{1}(u, v)-N_{1}\left(u^{\prime}, v^{\prime}\right)\right) \bar{U}\right\}, \\
& \left(|V|^{2}\right)_{t}-\left(|V|^{2}\right)_{x}=\Re 2\left\{i m U \bar{V}-i\left(N_{2}(u, v)-N_{2}\left(u^{\prime}, v^{\prime}\right)\right) \bar{V}\right\},
\end{aligned}
$$

For the nonlinear terms in the righthandsides of (4.1) and (4.2), we have following by direct computations.

Lemma 4.1. There exists a $c_{*}>0$ such that

$$
\left|\Re 2\left\{i m V \bar{U}-i\left(N_{1}(u, v)-N_{1}\left(u^{\prime}, v^{\prime}\right)\right) \bar{U}\right\}\right| \leq r_{1}(x, t)
$$

and

$$
\left|\Re 2\left\{i m U \bar{V}-i\left(N_{2}(u, v)-N_{2}\left(u^{\prime}, v^{\prime}\right)\right) \bar{V}\right\}\right| \leq r_{1}(x, t),
$$

where

$$
r_{1}(x, t)=m\left(|U(x, t)|^{2}+|V(x, t)|^{2}\right)+c_{*} r_{2}(x, x, t),
$$

$r_{2}(x, y, t)=|U(x, t)|^{2}\left(|v(y, t)|^{2}+\left|v^{\prime}(y, t)\right|^{2}\right)+\left(|u(x, t)|^{2}+\left|u^{\prime}(x, t)\right|^{2}\right)|V(y, t)|^{2}$.

To get the control on $(U, V)$ via (4.1) and (4.2), we introduce following functionals on $\Delta \cap \Omega$ for $(U, V)$ as in [25]. Here it is assume that $\Delta \cap \Omega \neq \emptyset$.

Definition 4.1. For $\Delta=\Delta\left(a, b, t_{0}\right)$ and $K_{1}>0, C_{1}>0$, define

$$
\begin{gathered}
L_{1}(t, \Delta)=L(t, U, \Delta)+K_{1} L(t, V, \Delta), \\
D_{1}(t, \Delta)=\int_{z_{a}(t)}^{b-t+t_{0}} r_{2}(x, x, t) d x, \\
Q_{1}(t, \Delta)=\iint_{z_{a}(t)<x<y<b-t+t_{0}} r_{2}(x, y, t) d x d y
\end{gathered}
$$

and

$$
F_{1}(t, \Delta)=L_{1}(t, \Delta)+C_{1} Q_{1}(t, \Delta)
$$

for $t \in I_{\Delta}$. Here $\Delta \cap \Omega \neq \emptyset$ with

$$
z_{a}(t)=\max \left\{a+t-t_{0}, z(t)\right\}
$$

$L(t, U, \Delta), L(t, V, \Delta)$ and $I_{\Delta}$ are given by Definition 3.1 in section [3. 
In addition we use the notations in Definition 3.2 for $(u, v)$, and use the following for $\left(u^{\prime}, v^{\prime}\right)$,

$$
L_{0}^{\prime}(t, \Delta)=L\left(t, u^{\prime}, \Delta\right)+L\left(t, v^{\prime}, \Delta\right)
$$

and

$$
\begin{gathered}
D_{0}^{\prime}(t, \Delta)=\int_{z_{a}(t)}^{b-t+t_{0}}\left|u^{\prime}(x, t)\right|^{2}\left|v^{\prime}(x, t)\right|^{2} d x \\
Q_{0}^{\prime}(t, \Delta)=\iint_{z_{a}(t)<x<y<b-t+t_{0}}\left|u^{\prime}(x, t)\right|^{2}\left|v^{\prime}(y, t)\right|^{2} d x d y
\end{gathered}
$$

for $t \in I_{\Delta}$, and

$$
r_{0}^{\prime}(x, t)=m\left(\left|u^{\prime}(x, t)\right|^{2}+\left|v^{\prime}(x, t)\right|^{2}\right)+8|\beta|\left|u^{\prime}(x, t)\right|^{2}\left|v^{\prime}(x, t)\right|^{2} .
$$

Moreover, (2.1) and (2.2) still hold for both $(u, v)$ and $\left(u^{\prime}, v^{\prime}\right)$, and Lemmas in Section 3 also hold for these two solution.

Now for any $T>0$, we can have the estimates on $F_{1}$ near the boundary $\Gamma_{B}$ as follows.

Lemma 4.2. Suppose that $\Delta \subset R^{1} \times[0, T]$ and $\Delta \cap \Gamma_{B} \neq \emptyset$. Then, there exist constants $\delta_{0}>0, K_{1}>0$ and $C_{1}>0$ such that if $L_{0}\left(t_{0}, \Delta\right) \leq \delta_{0}$ and $L_{0}^{\prime}\left(t_{0}, \Delta\right) \leq \delta_{0}$ then there holds the following,

$$
\frac{d}{d t} F_{1}(t, \Delta) \leq-D_{1}(t, \Delta)+\left[O(1)+C_{1} \Lambda_{1}(t, \Delta)+C_{1} \Lambda_{2}(t)\right] F_{1}(t, \Delta)
$$

for $t \in I_{\Delta}$ with $z(t) \neq a+t-t_{0}$, where

$$
\Lambda_{1}(t, \Delta)=4 m \delta_{0}+8|\beta|\left(D_{0}(t, \Delta)+D_{0}^{\prime}(t, \Delta)\right),
$$

and

$$
\Lambda_{2}(t)= \begin{cases}\left(1-z_{t}(t)\right)|\lambda(t)|^{2}\left(|v(z(t), t)|^{2}+\left|v^{\prime}(z(t), t)\right|^{2}\right), & \text { if } a+t-t_{0} \leq z(t) \\ 0, & \text { if } a+t-t_{0}>z(t) .\end{cases}
$$

Here the constants $K_{1}>1, \delta_{0}$ and $C_{1}$ depend only on $T$.

Proof. It suffices to prove lemma for two cases.

Case 1: The boundary $\Gamma_{B}$ and the right edge of $\Delta$ intersec at some point $\left(z\left(\tau_{1}\right), \tau_{1}\right)$, see Figure 4. Then $I_{\Delta}=\left[t_{0}, \tau_{1}\right]$, and $z_{a}(t)=z(t)$ for $t \in I_{\Delta}$. 
For $t \in\left(t_{0}, \tau_{1}\right)$, by Lemma 2.2 and Lemma 4.1, we use (4.1) and (2.2) for both $(u, v)$ and $\left(u^{\prime}, v^{\prime}\right)$ to derive that

$$
\begin{aligned}
& \frac{d}{d t} \iint_{z(t)<x<y<b-t+t_{0}}|U(x, t)|^{2}\left(|v(y, t)|^{2}+\left|v^{\prime}(y, t)\right|^{2}\right) d x d y \\
& \leq-\iint_{z(t)<x<y<b-t+t_{0}}\left(|U(x, t)|^{2}\right)_{x}\left(|v(y, t)|^{2}+\left|v^{\prime}(y, t)\right|^{2}\right) d x d y \\
& +\iint_{z(t)<x<y<b-t+t_{0}}|U(x, t)|^{2}\left(|v(y, t)|^{2}+\left|v^{\prime}(y, t)\right|^{2}\right)_{y} d x d y \\
& +\iint_{z(t)<x<y<b-t+t_{0}} r_{1}(x, t)\left(|v(y, t)|^{2}+\left|v^{\prime}(y, t)\right|^{2}\right) d x d y \\
& +\iint_{z(t)<x<y<b-t+t_{0}}|U(x, t)|^{2}\left(r_{0}(y, t)+r_{0}^{\prime}(y, t)\right) d x d y \\
& -\int_{z(t)}^{b-t+t_{0}}|U(x, t)|^{2}\left(\left|v\left(b-t+t_{0}, t\right)\right|^{2}+\left|v^{\prime}\left(b-t+t_{0}, t\right)\right|^{2}\right) d x \\
& -z_{t}(t) \int_{z(t)}^{b-t+t_{0}}|U(z(t), t)|^{2}\left(|v(y, t)|^{2}+\left|v^{\prime}(y, t)\right|^{2}\right) d y \\
& \leq-2 \int_{z(t)}^{b-t+t_{0}}|U(y, t)|^{2}\left(|v(y, t)|^{2}+\left|v^{\prime}(y, t)\right|^{2}\right) d y \\
& +\left(1-z_{t}(t)\right)|\lambda(t)|^{2}|V(z(t), t)|^{2} \int_{z(t)}^{b-t+t_{0}}\left(|v(y, t)|^{2}+\left|v^{\prime}(y, t)\right|^{2}\right) d y \\
& +\left(m L_{1}(t)+c_{*} D_{1}(t)\right) \int_{z(t)}^{b-t+t_{0}}\left(|v(y, t)|^{2}+\left|v^{\prime}(y, t)\right|^{2}\right) d y \\
& +\left(m L_{0}(t)+8|\beta| D_{0}(t)+m L_{0}^{\prime}(t)+8|\beta| D_{0}^{\prime}(t)\right) \int_{z(t)}^{b-t+t_{0}}|U(x, t)|^{2} d x,
\end{aligned}
$$


while by Lemma 2.2 and Lemma 4.1, we use (4.2) and (2.1) for both $(u, v)$ and $\left(u^{\prime}, v^{\prime}\right)$ to derive that

$$
\begin{aligned}
& \frac{d}{d t} \iint_{z(t)<x<y<b-t+t_{0}}\left(|u(x, t)|^{2}+\left|u^{\prime}(x, t)\right|^{2}\right)|V(y, t)|^{2} d x d y \\
\leq & -\iint_{z(t)<x<y<b-t+t_{0}}\left(|u(x, t)|^{2}+\left|u^{\prime}(x, t)\right|^{2}\right)_{x}|V(y, t)|^{2} d x d y \\
& +\iint_{z(t)<x<y<b-t+t_{0}}\left(|u(x, t)|^{2}+\left|u^{\prime}(x, t)\right|^{2}\right)\left(|V(y, t)|^{2}\right)_{y} d x d y \\
& +\iint_{z(t)<x<y<b-t+t_{0}}\left(r_{0}(x, t)+r_{0}^{\prime}(x, t)\right)|V(y, t)|^{2} d x d y \\
& +\iint_{z(t)<x<y<b-t+t_{0}}\left(|u(x, t)|^{2}+\left|u^{\prime}(x, t)\right|^{2}\right) r_{1}(y, t) d x d y \\
& -z_{t}(t) \int_{z(t)}^{b-t+t_{0}}\left(|u(z(t), t)|^{2}+\left|u^{\prime}(z(t), t)\right|^{2}\right)|V(y, t)|^{2} d y \\
& -\int_{z(t)}^{b-t+t_{0}}\left(|u(x, t)|^{2}+\left|u^{\prime}(x, t)\right|^{2}\right)\left|V\left(b-t+t_{0}, t\right)\right|^{2} d x \\
\leq & -2 \int_{z(t)}^{b-t+t_{0}}\left(|u(x, t)|^{2}+\left|u^{\prime}(x, t)\right|^{2}|V(x, t)|^{2}\right) d x \\
+ & \left(1-z_{t}(t)\right)\left(|u(z(t), t)|^{2}+\left|u^{\prime}(z(t), t)\right|^{2}\right) \int_{z(t)}^{b-t+t_{0}}|V(x, t)|^{2} d x \\
+ & \left(m L_{1}(t)+c_{*} D_{1}(t)\right) \int_{z(t)}^{b-t+t_{0}}\left(|u(x, t)|^{2}+\left|u^{\prime}(x, t)\right|^{2}\right) d x \\
+ & \\
+ &
\end{aligned}
$$

Collecting these two inequalities, we have the estimates on $Q_{1}$ as follows,

$$
\begin{aligned}
\frac{d}{d t} Q_{1}(t) \leq & {\left[-2+c_{*}\left(L_{0}(t)+L_{0}^{\prime}(t)\right)\right] D_{1}(t)+\left[q_{1}(t)+q_{2}(t)\right] L_{1}(t) } \\
& +\left(1-z_{t}(t)\right)|\lambda(t)|^{2}|V(z(t), t)|^{2}\left(L_{0}(t)+L_{0}^{\prime}(t)\right)
\end{aligned}
$$

where

$$
q_{1}(t)=2 m\left(L_{0}(t)+L_{0}^{\prime}(t)\right)+8|\beta|\left(D_{0}(t)+D_{0}^{\prime}(t)\right)
$$

and

$$
q_{2}(t)=\left(1-z_{t}(t)\right)|\lambda(t)|^{2}\left(|v(z(t), t)|^{2}+\left|v^{\prime}(z(t), t)\right|^{2}\right) .
$$


For the functional $L_{1}$, by (4.1) and by Lemma 4.1, we have

$$
\begin{aligned}
\frac{d}{d t} L(t, U) \leq & -\left|U\left(b-t+t_{0}, t\right)\right|^{2}-z_{t}(t)|U(z(t), t)|^{2} \\
& -\int_{z(t)}^{b-t+t_{0}}\left(|U(x, t)|^{2}\right)_{x} d x+\int_{z(t)}^{b-t+t_{0}} r_{1}(x, t) d x \\
\leq & \left(1-z_{t}(t)\right)|\lambda(t)|^{2}|V(z(t), t)|^{2}+\int_{z(t)}^{b-t+t_{0}} r_{1}(x, t) d x
\end{aligned}
$$

while by (4.2) and by Lemma 4.1, we have

$$
\begin{aligned}
\frac{d}{d t} L(t, V) \leq & -\left|V\left(b-t+t_{0}, t\right)\right|^{2}-z_{t}(t)|V(z(t), t)|^{2} \\
& +\int_{z(t)}^{b-t+t_{0}}\left(|V(x, t)|^{2}\right)_{x} d x+\int_{z(t)}^{b-t+t_{0}} r_{1}(x, t) d x \\
\leq & -\left(1+z_{t}(t)\right)|V(z(t), t)|^{2}+\int_{z(t)}^{b-t+t_{0}} r_{1}(x, t) d x
\end{aligned}
$$

Then we have the following estimate on $L_{1}$,

$$
\begin{aligned}
\frac{d}{d t}\left(L(t, U)+K_{1} L(t, V)\right) \leq & {\left[\left(1-z_{t}(t)\right)|\lambda(t)|^{2}-K_{1}\left(1+z_{t}(t)\right)\right]|V(z(t), t)|^{2} } \\
& +\left(1+K_{1}\right) \int_{z(t)}^{b-t+t_{0}} r_{1}(x, t) d x \\
\leq & -2|V(z(t), t)|^{2}+\left(1+K_{1}\right)\left(m L_{1}(t)+c_{*} D_{1}(t)\right) .
\end{aligned}
$$

Here the constant $K_{1}>1$ is chosen so that

$$
\max _{t \in[0, T]}\left[\left(1-z_{t}(t)\right)|\lambda(t)|^{2}-K_{1}\left(1+z_{t}(t)\right)\right]<-2 .
$$

Now, with the above estimates on $Q_{1}$ and $L_{1}$, we use Lemma 3.2 to derive the following,

$$
\begin{aligned}
\frac{d}{d t} F_{1}(t) \leq & \left\{\left(1+K_{1}\right) c_{*}+\left[-2+c_{*}\left(L_{0}(t)+L^{\prime}(t)\right)\right] C_{1}\right\} D_{1}(t) \\
& +\left[-2+C_{1}\left(1-z_{t}(t)\right)|\lambda(t)|^{2}\left(L_{0}(t)+L_{0}^{\prime}(t)\right)\right]|V(z(t), t)|^{2} \\
& +\left[\left(1+K_{1}\right) m+C_{1}\left(q_{1}(t)+q_{2}(t)\right)\right] L_{1}(t) \\
\leq & -D_{1}(t)+\left[\left(1+K_{1}\right) m+C_{1}\left(\Lambda_{1}(t)+\Lambda_{2}(t)\right)\right] L_{1}(t)
\end{aligned}
$$

for $L_{0}\left(t_{0}\right) \leq \delta_{0}$ and $L_{0}^{\prime}\left(t_{0}\right) \leq \delta_{0}$, where we choose $\delta_{0}>0$ and $C_{1}>0$ so that

$$
-2+2 c_{*} \delta_{0}<-1, \quad\left(1+K_{1}\right) c_{*}-C_{1}<-1
$$

and

$$
-2+2 C_{1} \delta_{0} \max _{0 \leq t \leq T}\left(1-z_{t}(t)\right)|\lambda(t)|^{2}<-1
$$


Therefore (4.3) is proved for Case 1 .

Case 2: The boundary $\Gamma_{B}$ and the left edge of $\Delta$ intersect at $\left(z\left(\tau_{2}\right), \tau_{2}\right)$. Then, $I_{\Delta}=\left[t_{0}, t_{0}+\frac{b-a}{2}\right]$, and $z_{a}(t)=z(t)$ for $t_{0} \leq t \leq \tau_{2}, z_{a}(t)=a-t_{0}+t$ for $\tau_{2} \leq t \leq t_{0}+\frac{b-a}{2}$. The proof can be carried out in the same way as in Case 1 for $t \neq \tau_{2}$. Thus the proof is complete.

Remark 4.1. For the case that $\Delta \subset \Omega(T)$, we have similar estimates on $F_{1}$ without boundary terms, see [25] for the proof, where only $D_{1}(t, \Delta)$ makes contribution to the control on $F_{1}$. For the case that $\Delta \cap \Gamma_{B} \neq \emptyset$, both $Q_{1}(t, \Delta)$ and $L(t, V, \Delta)$ are needed to give the control on $F_{1}$.

As conclusion of the above argument, we get the stability result for smooth solutions for any $T>0$.

Proposition 4.1. Suppose that $\Delta \subset R^{1} \times[0, T]$ with $b>z\left(t_{0}\right)$, and suppose that $L_{0}\left(t_{0}, \Delta\right) \leq \delta_{0}, L_{0}^{\prime}\left(t_{0}, \Delta\right) \leq \delta_{0}$. Then for $t \in I_{\Delta}$, there holds the following

$$
\begin{aligned}
& \int_{z_{a}(t)}^{b+t_{0}-t}\left(\left|u(x, t)-u^{\prime}(x, t)\right|^{2}+\left|v(x, t)-v^{\prime}(x, t)\right|^{2}\right) d x \\
\leq & C_{4} \int_{\max \left\{z\left(t_{0}\right), a\right\}}^{b}\left(\left|u\left(x, t_{0}\right)-u^{\prime}\left(x, t_{0}\right)\right|^{2}+\left|v\left(x, t_{0}\right)-v^{\prime}\left(x, t_{0}\right)\right|^{2}\right) d x,
\end{aligned}
$$

and

$$
\begin{aligned}
& \iint_{\Delta \cap \Omega}\left(\left|u v-u^{\prime} v^{\prime}\right|^{2}\right) d x d t \\
\leq & C_{4} \int_{\max \left\{z\left(t_{0}\right), a\right\}}^{b}\left(\left|u\left(x, t_{0}\right)-u^{\prime}\left(x, t_{0}\right)\right|^{2}+\left|v\left(x, t_{0}\right)-v^{\prime}\left(x, t_{0}\right)\right|^{2}\right) d x \\
& \iint_{\Delta \cap \Omega}\left(\left|u \bar{v}-u^{\prime} \overline{v^{\prime}}\right|^{2}\right) d x d t \\
\leq & C_{4} \int_{\max \left\{z\left(t_{0}\right), a\right\}}^{b}\left(\left|u\left(x, t_{0}\right)-u^{\prime}\left(x, t_{0}\right)\right|^{2}+\left|v\left(x, t_{0}\right)-v^{\prime}\left(x, t_{0}\right)\right|^{2}\right) d x
\end{aligned}
$$

Here the constant $C_{4}$ depends only on $T$ and $E_{0}$.

Proof. It suffices to prove lemma for two cases.

Case 1: $a<z\left(t_{0}\right)<b$, that is, $\Delta \cap \Gamma_{B} \neq \emptyset$. Then taking the integral of (3.4) in Lemma 3.4 over $I_{\Delta}$, we have

$$
\begin{gathered}
\int_{I_{\Delta}}\left(D_{0}(t, \Delta)+D_{0}^{\prime}(t, \Delta)+\left|v\left(z_{a}(t), t\right)\right|^{2}+\left|v^{\prime}\left(z_{a}(t), t\right)\right|^{2}\right) d t \\
\leq O(1)\left(L_{0}\left(t_{0}, \Delta\right)+L_{0}^{\prime}\left(t_{0}, \Delta\right)\right)+O(1) \delta_{0} T
\end{gathered}
$$

which leads to

$$
\int_{I_{\Delta}}\left(\Lambda_{1}(t, \Delta)+\Lambda_{2}(t, \Delta)\right) d t \leq C(T)
$$


for some constant $C(T)>0$ depending on $T$.

Therefore, we use Lemma 4.2 to deduce that

$$
\begin{aligned}
F_{1}(t) & \leq \exp \left(\int_{I_{\Delta}}\left[O(1)+C_{1} \Lambda_{1}(s, \Delta)+C_{1} \Lambda_{2}(s, \Delta)\right] d s\right) F_{1}\left(t_{0}\right) \\
& \leq C^{\prime}(T) \int_{z\left(t_{0}\right)}^{b}\left(\left|u\left(x, t_{0}\right)-u^{\prime}\left(x, t_{0}\right)\right|^{2}+\left|v\left(x, t_{0}\right)-v^{\prime}\left(x, t_{0}\right)\right|^{2}\right) d x
\end{aligned}
$$

for $t \in I_{\Delta}$, and

$$
\begin{aligned}
\int_{I_{\Delta}} D_{1}(t, \Delta) d t & \leq F_{1}\left(t_{0}\right) \\
& +\left(\int_{I_{\Delta}}\left[O(1)+C_{1} \Lambda_{1}(s, \Delta)+C_{1} \Lambda_{2}(s, \Delta)\right] d s\right) T \max _{t_{0} \leq t \leq t_{0}+\frac{b-a}{2}} F_{1}(t) \\
& \leq F_{1}\left(t_{0}\right)+C^{\prime \prime}(T) \max _{t_{0} \leq t \leq t_{0}+\frac{b-a}{2}} F_{1}(t),
\end{aligned}
$$

which lead to the result for Case 1 . Here the constants $C^{\prime}(T)$ and $C^{\prime \prime}(T)$ depend only on $T$.

Case 2: $z\left(t_{0}\right)<a$, that is, $\Delta \cap \Gamma_{B}=\emptyset$ and $\Delta \subset \Omega(T)$. Then $z_{a}(t)=a+t-t_{0}$. The result for this case has been proved in [25], and its proof can be carried out in the same way as above. Therefore the proof is complete.

\section{Convergence of Global Classical solutions}

Choose a sequence of smooth functions

$$
\left(u_{0}^{(k)}, v_{0}^{(k)}\right) \in C_{c}^{\infty}(0, \infty), \quad k=1,2, \cdot,
$$

such that

$$
\left(u_{0}^{(k)}, v_{0}^{(k)}\right) \rightarrow\left(u_{0}, v_{0}\right) \quad \text { in } L_{l o c}^{2}(0, \infty)
$$

as $m \rightarrow \infty$. Theorem 1.1 implies that there is a sequence of classical solutions, $\left(u^{(k)}, v^{(k)}\right) \in C^{1}(\bar{\Omega}), k=1,2, \cdots$, to (1.1), which satisfy boundary condition (1.3) and take $\left(u_{0}^{(k)}, v_{0}^{(k)}\right)$ as their initial data respectively. And $\operatorname{supp}\left(u^{(k)}(\cdot, t), v^{(k)}(\cdot, t)\right)$ has bounded support in $R^{1}$ for any $t \geq 0$ and $k \geq 0$.

We consider the convergence of $\left\{\left(u^{(k)}, v^{(k)}\right)\right\}_{k=0}^{\infty}$ on $\Delta(-A, A, 0) \cap \Omega$ for any $A>0$. To this end, we first give the estimate on $L^{2}$ norm of solution over small interval $[a, b] \cap[z(t), A-t]$ for any $a$ and $b$.

Lemma 5.1. There is a constant $r>0$ such that if $0<b-a \leq 4 r$ and $b \leq A$ then

$$
\sup _{k \geq 1} \int_{\max \{z(t), a\}}^{\min \{b, A-t\}}\left(\left|u^{(k)}(x, t)\right|^{2}+\left|v^{(k)}(x, t)\right|^{2}\right) d x \leq \delta_{0}
$$

for $t \in[0, A]$ with $z(t) \leq b$. 
Proof.It is obvious that

$$
\lim _{k \rightarrow \infty} \int_{0}^{A}\left(\left|u_{0}^{(k)}-u_{0}\right|^{2}+\left|v_{0}^{(k)}-v_{0}\right|^{2}\right) d x=0 .
$$

As in [25], we choose $r>0$ such that

$$
\exp \left(m A+8|\beta| j_{0}\right)\left(\int_{\max \{z(0), a\}}^{b}\left(\left|u_{0}^{(k)}(x)\right|^{2}+\left|v_{0}^{(k)}(x)\right|^{2}\right) d x+m j_{0}(b-a)\right) \leq \frac{\delta_{0}}{8}
$$

and

$$
\exp \left(2 m A+16|\beta| j_{0}\right)\left(\int_{\max \{z(0), a\}}^{b}\left|v_{0}^{(k)}(2 p(x)+x)\right|^{2} d x+m j_{0}(b-a)\right) \leq \frac{\delta_{0}}{8}
$$

for $|b-a| \leq 4 r$ and for $k=0,1,2 \cdots$. Here for simplification $\left(u_{0}^{(0)}, v_{0}^{(0)}\right)=$ $\left(u_{0}, v_{0}\right)$.

Then with the pointwise estimates along the characteristics in Lemma 2.6 and Lemma 2.8, we can deduce the desired result. The proof is complete.

Now application of Proposition 4.1 and Lemma 5.1 to any pair of smooth solutions $\left(u^{(k)}, v^{(k)}\right)$ and $\left(u^{(n)}, v^{(n)}\right)$ gives the following.

Lemma 5.2. Suppose that $\Delta(a, b, \tau) \subset \Delta(-A, A, 0)$ with $0<b-a \leq 4 r$ and $\Delta(a, b, \tau) \cap \Omega \neq \emptyset$. Then there exists a constant $C(A)>0$ such that

$$
\begin{aligned}
& \iint_{\Delta(a, b, \tau) \cap \Omega}\left(\left|u^{(k)}-u^{(n)}\right|^{2}+\left|v^{(k)}-v^{(n)}\right|^{2}\right) d x d t \\
+ & \iint_{\Delta(a, b, \tau) \cap \Omega}\left(\left|u^{(k)} v^{(k)}-u^{(n)} v^{(n)}\right|^{2}+\left|u^{(k)} \overline{v^{(k)}}-u^{(n)} \overline{v^{(n)}}\right|^{2}\right) d x d t \\
\leq & C(A) \int_{\max (z(0), a)}^{b}\left(\left|u^{(k)}(x, \tau)-u^{(n)}(x, \tau)\right|^{2}+\left|v^{(k)}(x, \tau)-v^{(n)}(x, \tau)\right|^{2}\right) d x
\end{aligned}
$$

for any $k \geq 1$ and $n \geq 1$. Here the constant $C(A)$ depends only on $A$ and $E_{0}$; the constant $r>0$ is given by Lemma 5.1.

In the next, we prove the convergence of $\left\{\left(u^{(k)}, v^{(k)}\right)\right\}_{k=0}^{\infty}$ on $\Delta(-A, A, 0) \cap \Omega$ by the induction step as follows.

Denote

$$
\Omega(A, \tau)=\Delta(-A, A, 0) \cap \Omega \cap\{(x, t) \mid 0 \leq t \leq \tau\}, \quad 0 \leq \tau \leq A .
$$

Lemma 5.3. Suppose that

$$
\lim _{m, n \rightarrow \infty} \iint_{\Omega(A, \tau)}\left(\left|u^{(m)}-u^{(n)}\right|^{2}+\left|v^{(m)}-v^{(n)}\right|^{2}\right) d x d t=0,
$$




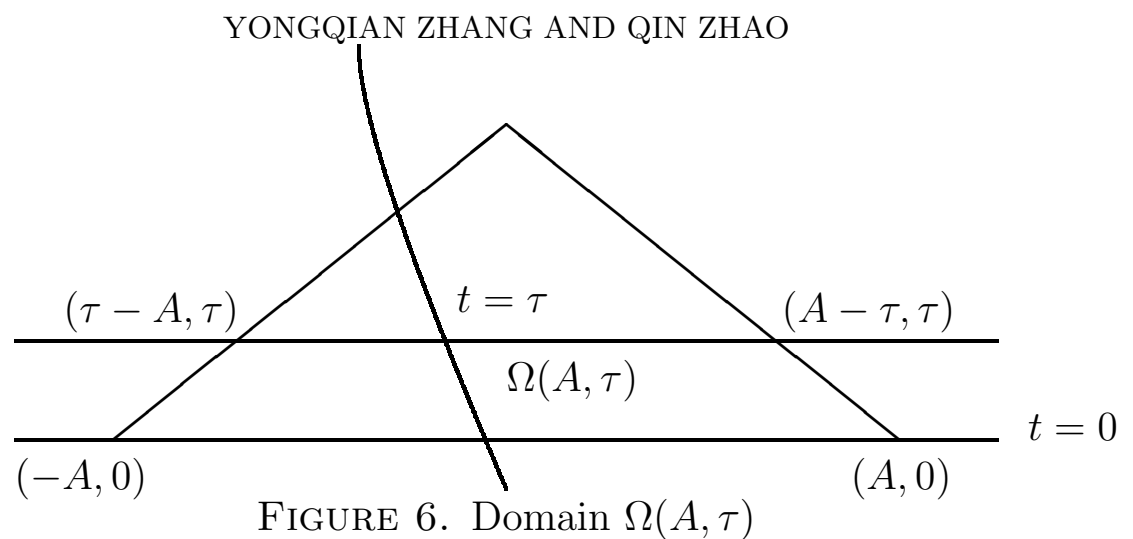

$$
\lim _{m, n \rightarrow \infty} \iint_{\Omega(A, \tau)}\left(\left|u^{(m)} v^{(m)}-u^{(n)} v^{(n)}\right|^{2}\right) d x d t=0
$$

and

$$
\lim _{m, n \rightarrow \infty} \iint_{\Omega(A, \tau)}\left(\left|u^{(m)} \overline{v^{(m)}}-u^{(n)} \overline{v^{(n)}}\right|^{2}\right) d x d t=0
$$

for $\tau \in[0, A-r]$. Then

$$
\begin{gathered}
\lim _{m, n \rightarrow \infty} \iint_{\Omega(A, \tau+r)}\left(\left|u^{(m)}-u^{(n)}\right|^{2}+\left|v^{(m)}-v^{(n)}\right|^{2}\right) d x d t=0, \\
\lim _{m, n \rightarrow \infty} \iint_{\Omega(A, \tau+r)}\left(\left|u^{(m)} v^{(m)}-u^{(n)} v^{(n)}\right|^{2}\right) d x d t=0
\end{gathered}
$$

and

$$
\lim _{m, n \rightarrow \infty} \iint_{\Omega(A, \tau+r)}\left(\left|u^{(m)} \overline{v^{(m)}}-u^{(n)} \overline{v^{(n)}}\right|^{2}\right) d x d t=0 .
$$

Here $r$ is given in Lemma 5.1 .

Proof. We choose a finite number of subintervals, $\left[a_{j}, b_{j}\right], j=1,2, \cdots, J$, with $\left[a_{j}, b_{j}\right] \cap[\max \{\tau-A, z(\tau)\}, A-\tau] \neq \emptyset$ and $b_{j}-a_{j}=4 r$, such that

$$
\overline{\Omega(A, \tau+r) \backslash \Omega(A, \tau)} \subset \cup_{j=1}^{J} \Delta\left(a_{j}, b_{j}, \tau^{\prime}\right),
$$

where $\tau^{\prime}=\tau-\frac{r}{8}$, and $J \leq \frac{4 A}{r}+1$.

For $1 \leq j \leq J$, by Proposition 4.1 and Lemma 5.1, we have

$$
\begin{gathered}
\lim _{m, n \rightarrow \infty} \iint_{\Delta\left(a_{j}, b_{j}, \tau^{\prime}\right) \cap \Omega}\left(\left|u^{(m)}-u^{(n)}\right|^{2}+\left|v^{(m)}-v^{(n)}\right|^{2}\right) d x d t=0, \\
\lim _{m, n \rightarrow \infty} \iint_{\Delta\left(a_{j}, b_{j}, \tau^{\prime}\right) \cap \Omega}\left(\left|u^{(m)} v^{(m)}-u^{(n)} v^{(n)}\right|^{2}\right) d x d t=0
\end{gathered}
$$

and

$$
\lim _{m, n \rightarrow \infty} \iint_{\Delta\left(a_{j}, b_{j}, \tau^{\prime}\right) \cap \Omega}\left(\left|u^{(m)} \overline{v^{(m)}}-u^{(n)} \overline{v^{(n)}}\right|^{2}\right) d x d t=0 .
$$


Therefore we have the convergence of the sequences $\left\{\left(u^{(m)}, v^{(m)}\right)\right\}_{m=1}^{\infty},\left\{u^{(m)} v^{(m)}\right\}_{m=1}^{\infty}$ and $\left\{u^{(m)} \overline{v^{(m)}}\right\}_{m=1}^{\infty}$ in $L^{2}(\Omega(A, \tau+r))$ respectively. The proof is complete.

Now we have the following convergence result.

Proposition 5.1. There exists $a(u, v) \in L_{l o c}^{2}(\Omega)$ such that

$$
\lim _{m \rightarrow \infty}\left\|\left(u^{(m)}, v^{(m)}\right)-(u, v)\right\|_{L^{2}(\Delta(-A, A, 0) \cap \Omega)}=0
$$

and

$$
\lim _{m \rightarrow \infty}\left(\left\|u^{(m)} v^{(m)}-u v\right\|_{L^{2}(\Delta(-A, A, 0) \cap \Omega)}+\left\|u^{(m)} \overline{v^{(m)}}-u \bar{v}\right\|_{L^{2}(\Delta(-A, A, 0) \cap \Omega)}\right)=0
$$

for any $A>0$.

Proof. With the induction steps given by Lemma 5.3, we have

$$
\begin{gathered}
\lim _{m, n \rightarrow \infty} \iint_{(\Delta(-A, A, 0) \cap \Omega)}\left(\left|u^{(m)}-u^{(n)}\right|^{2}+\left|v^{(m)}-v^{(n)}\right|^{2}\right) d x d t=0, \\
\lim _{m, n \rightarrow \infty} \iint_{(\Delta(-A, A, 0) \cap \Omega)}\left(\left|u^{(m)} v^{(m)}-u^{(n)} v^{(n)}\right|^{2}\right) d x d t=0
\end{gathered}
$$

and

$$
\lim _{m, n \rightarrow \infty} \iint_{(\Delta(-A, A, 0) \cap \Omega)}\left(\left|u^{(m)} \overline{v^{(m)}}-u^{(n)} \overline{v^{(n)}}\right|^{2}\right) d x d t=0,
$$

for any $A>0$. These lead to the desired result. The proof is complete.

\section{Proof of main Results on Strong SOlutions}

In the same way as in the proof of Lemma 5.3 and Proposition 5.1, we can prove the following.

Proposition 6.1. Suppose that $\left\{u_{j}^{(m)}, v_{j}^{(m)}\right\}_{m=1}^{\infty}, j=1,2$, are two sequences of classical solution to (1.1) satisfy boundary condition (1.3) with the following,

$$
\lim _{m \rightarrow \infty} \int_{0}^{M}\left(\left|u_{1}^{(m)}(x, 0)-u_{2}^{(m)}(x, 0)\right|^{2}+\left|v_{1}^{(m)}(x, 0)-v_{2}^{(m)}(x, 0)\right|^{2}\right) d x=0
$$

for some $M>0$. Then,

$$
\lim _{m \rightarrow \infty} \iint_{\Delta(-M, M, 0) \cap \Omega}\left(\left|u_{1}^{(m)}-u_{2}^{(m)}\right|^{2}+\left|v_{1}^{(m)}-v_{2}^{(m)}\right|^{2}\right) d x d t=0 .
$$

Proof of Theorem 1.3. The existence of solution $(u, v)$ is proved by Proposition 5.1. Moreover, $(u, v)$ satisfies (1.9) and (2.6).

To prove the uniqueness, let $\left(u_{j}, v_{j}\right), j=1,2$, be two strong solutions to (1.1.1.3), and let $\left(u_{j}^{(m)}, v_{j}^{(m)}\right), j=1,2$ be two sequences of classical solutions to (1.1) with boundary condition (1.3), which are convergent to $\left(u_{j}, v_{j}\right), j=1,2$, respectively in $L_{l o c}^{2}(\Omega)$. Moreover, the initial data $\left(u_{j}^{(m)}(x, 0), v_{j}^{(m)}(x, 0)\right)$ are assumed to be convergent to $\left(u_{0}, v_{0}\right)$ for $j=1,2$. 
Then by Proposition 6.1, we have

$\lim _{m \rightarrow \infty} \iint_{\Delta(-A, A, 0) \cap \Omega}\left(\left|u_{1}^{(m)}(x, 0)-u_{2}^{(m)}(x, 0)\right|^{2}+\left|v_{1}^{(m)}(x, 0)-v_{2}^{(m)}(x, 0)\right|^{2}\right) d x=0$,

which yields that

$$
\left(u_{1}, v_{1}\right)(x, t)=\left(u_{2}, v_{2}\right)(x, t), \quad \text { a.e. }(x, t) \in \Delta(-A, A, 0) \cap \Omega .
$$

This leads to the uniqueness of the strong solution. The proof is complete.

Proof of Theorem 1.4. Indeed the results hold for the classical solutions. Then by taking the limit, we can prove the result still hold for the strong solution. The proof is complete.

\section{ACKNOWLEDGEMENT}

This work was partially supported by NSFC Project 11421061 and by the 111 Project B08018.

\section{REFERENCES}

[1] S. Alinhac, Blowup for nonlinear hyperbolic equations, Birkhauser Boston, Inc., Boston, 1995.

[2] A. Bachelot, Global Cauchy problem for semilinear hyperboloc systems with nonlocal interactions. Applications to Dirac equations, J. Math. Pures. Appl. 86 (2006), 201-236.

[3] J. M. Bony, Solution globales bornées pour les modèles discrets de l'équation de Boltzmann, en dimension 1 d'espace, Journées "Equations aux derivées partielles" (Saint Jean de Monts, 1987), Exp. No XVI, 10pp., École Polytech., Palaiseau, 1987.

[4] N. Bournaveas and G. E. Zouraris, Theory and numerical approximations for a nonlinear $1+1$ Dirac system, ESAIM: Math. Model. Num. Analysis 46 (4) (2012), 841-874.

[5] P. Bouvier and C. Gérard, Hawking effect for toy model of interacting Fermions, Ann. Heri. Poincaré 16 (5) (2015), 1191-1230.

[6] F. Cacciafesta, Global small solutions to the critical radial Dirac equation with potential, Nonlinear Analysis 74 (2011), 6060-6073.

[7] T. Candy, Global existence for an $L^{2}$ critical nonlinear Dirac equation in one dimension, Adv. Differential Equations 16 No. 7-8 (2011), 643-666.

[8] C. M. Dafermos, Hyperbolic Conservation Laws in Continuum Physics, Springer-Verlag, Berlin, 2010.

[9] V. Delgado, Global solution of the Cauchy problem for the (classical) coupled MaxwellDirac and other nonlinear Dirac equations, Proc. Amer. Math. Soc. 69(2)(1978), 289296.

[10] J. P. Dias and M. Figueira, Remarque sur le problème de Cauchy pour une equation de Dirac non linéaire avec masse nulle, Portucaliae Math. 45(4) (1988), 327-335.

[11] M. Escobedo and L. Vega, A semilinear Dirac equation in $H^{s}\left(R^{3}\right)$ for $s>1$, SIAM J. Math. Anal. 28(2) (1997), 338-362.

[12] M. J. Esteban, M. Lewin and E. Séré, Variational methods in relativistic quantum mechanics, Bulletin A.M.S. 45 No. 4 (2008), 535-598.

[13] J. Glimm, Solution in the large for nonlinear systems of conservation laws, Comm. Pure Appl. Math. 18(1965), 695-715. 
[14] D. J. Gross and A. Neveu, Dynamical symmetry breaking in asymptotically free field theories, Phys. Rev. D 10 (1974), 3235-3253.

[15] S-Y Ha and A. E. Tzavaras, Lyapunov functionals and $L^{1}$-stability for discrete velocity Boltzmann equations, Commun. Math. Phys. 239 (2003), 65-92.

[16] H. Huh, Global solutions to Gross-Neveu equation, Lett. Math. Phys. 103 (8)(2013), 927-931.

[17] Mizohata, The Theory of Partial Differential Equations, Cambridge University Press, New York, 1973.

[18] I.P. Naumkin, Cubic nonlinear Dirac equation in a quarter plane, J. Math. Anal. Appl. 434 (2) (2016), 1633-1664.

[19] I. P. Naumkin, Initial boundary value problem for the one dimensional Thirring model, J. Differential Equations 261 (8) (2016), 4486-4523.

[20] D. Pelinovsky, Survey on global existence in the nonlinear Dirac equations in one dimension, in: Harmonic analysis and nonlinear partial differential equations (edited by T. Ozawa and M. Sugimoto), RIMS Kôkyûroku Bessatsu, B26 (2011), 37-50.

[21] S. Selberg, Global existence in the critical space for the Thirring and Gross-neveu models coupled with the electromagnetic field, Disrete Contin. Dyn. Syst. 38 (5) (2018), 25552569.

[22] L. Tartar, From Hyperbolic Systems to Kinetic Theory, Springer 2008.

[23] W.E. Thirring, A soluble relativistic field theory, Ann. Phys. 3(1958), 91-112.

[24] Y. Zhang, Global strong solution to a nonlinear Dirac type equation in one dimension, Nonlinear Analysis: Theory, Method and Applications 80 (2013), 150-155.

[25] Y. Zhang and Q. Zhao, Global solution to nonlinear Dirac equation for Gross-Neveu model in $1+1$ dimensions, Nonlinear Analysis: Theory, Method and Applications 118 (2015), 82-96.

[26] Y. Zhou, Uniqueness of weak solutions in $1+1$ dimensional wave maps, Math. Z. 232(1999), 707-719.

Yongqian Zhang: School of Mathematical Sciences, Fudan University, Shanghai 200433, P.R.China

E-mail address: yongqianz@fudan.edu.cn

Qin Zhao : School of Mathematical Sciences, Shanghai Jiao Tong UniVersity, Shanghai 200240, P.R.China

E-mail address: zhao@sjtu.edu.cn 